\title{
Review of: "The impact of shielding during the COVID-19 pandemic on mental health: Evidence from the English Longitudinal Study of Ageing"
}

\author{
M.Soledad Herrera ${ }^{1}$ \\ 1 Pontifical Catholic University of Chile
}

Potential competing interests: The author(s) declared that no potential competing interests exist.

It is a clear and well-executed paper. Nevertheless, I have some observations.

"Covariates. Our analyses controlled for a wide range of demographic, socio-economic characteristics, health, and social support characteristics". The variables of the study do not measure "social support characteristics". I think that the best concept is "isolation" or "social contacts". Social support refers to some kind of interchange of services and isolation refers to having social contacts.

Sometimes you talk about two waves of the COVID Data ("We used the most recent pre-pandemic data (wave 9, collected in 2018/19) and the two waves of the COVID-19 sub-study (collected in June/July and November/December 2020 respectively)", and afterwards you talk about “April 2020, June/July 2020 and November/December 2020". Please check it through the article.

Conclusion and discussion. Bibliographical references are missing in the discussion.

In the limitations, a reference should be made to how the COVID panel follow-up could be biased by selective non-response. For example, due to the change in the methodology for applying the questionnaire. 